processes were assessed using Pearson correlation. Framework and content analysis were used to qualitatively explore PHCT perceptions of teamwork effectiveness.

Results Mean PA during match congestion was 78.1\% (95\% BCa: 76.2, 80.4) compared with 84.2 (95\% BCa: 80.6, 87.3) during uncongested periods. There were significant associations between match frequency and PA $(r=-0.68$; 95\% BCa: $0.32,0.93 ; p=0.008)$ and PHCT processes and PA $(r=0.53 ; 95 \%$ BCa: 0.09, 0.89; $\mathrm{p}=0.035)$. Having more PHCT meetings $(r=0.46$; BCa 95\%: $0.22,0.82 ; p=$ $0.048)$ and greater satisfaction with those meetings $(r=$ 0.41; BCa 95\%: 0.04, 0.07; $p=0.043$ ) were associated with higher PA, irrespective of match frequency. During match congestion the PHCT reported issues relating to resources and task co-ordination that negatively impacted their processes.

Conclusion The structure and processes adopted by a PHCT in professional football are related to PA, reflecting the influence of teamwork effectiveness. These findings have implications for injury prevention and management in professional football.

\section{ATTITUDES, BELIEFS, AND BEHAVIOUR TO THE ADDUCTOR STRENGTHENING PROGRAMME IN MALE PROFESSIONAL FOOTBALL: SUCCESSFULLY ADOPTED, BUT USUALLY MODIFIED}

${ }^{1}$ Joakim Stensø, ${ }^{2}$ Thor Einar Andersen, ${ }^{2}$ Joar Harøy. ${ }^{1}$ Norwegian School of Sport Sciences, Oslo, Norway; ${ }^{2}$ Oslo Sports Trauma Research Center, Department of Sports Medicine, Norwegian School of Sport Sciences, Oslo, Norway

\subsection{6/bjsports-2021-IOC.389}

Background The Adductor Strengthening Programme (ASP) is the first groin specific prevention programme proven to reduce the risk of groin problems in male football. Widespread dissemination of the programme and its preventative effect is recommended, however, successful implementation require researchers acquiring comprehensive knowledge of the implementation context. Using the Reach Effectiveness Adoption Implementation Maintenance (RE-AIM) framework is recommended for this procedure.

Objectives First, to investigate delivery agents' attitudes, beliefs, and behaviour regarding the ASP using the RE-AIM framework. Second, to present a 'best practice' protocol based on the reported usage of the ASP in a professional team setting.

Design Descriptive cross-sectional.

Setting Norwegian male professional football teams.

Participants The primary delivery agent of injury prevention exercise programmes in each team $(n=32)$.

Intervention Survey using a pilot tested questionnaire.

Results Twenty-nine (91\%) participants responded. All respondents (100\%) were familiar with the ASP and its potential to mitigate the burden of groin problems. All delivery agents (100\%) adopted the ASP, however, only $10 \%$ used it in accordance with the evidence-based protocol. The main modifications were that players in $72 \%$ of the teams were instructed to perform a non-progressive number of repetitions during pre-season, and $86 \%$ of the teams performed more sets, but fewer repetitions per set during in-season. In total, $97 \%$ of delivery agents planned to continue using the
ASP in the subsequent season. The two most stated reasons for using the ASP, were first, its documented injury preventive effect and second, that it doesn't require any additional equipment.

Conclusion The delivery agents had positive attitudes and beliefs to the ASP. Moreover, they widely adopted and planned to maintain its usage in the next season. Most of the delivery agents modified the original ASP protocol, which warrant further investigations.

\section{THE ASSOCIATION BETWEEN COVID-19 AND PHYSICAL PERFORMANCE IN PROFESSIONAL FOOTBALL PLAYERS: A PROSPECTIVE COHORT STUDY}

${ }^{1}$ Evi Wezenbeek, ${ }^{1}$ Sander Denolf, ${ }^{2}$ Jan Bourgois, ${ }^{3}$ Renaat Philippaerts, ${ }^{4}$ Bram De Winne, ${ }^{1}$ Erik Witvrouw, ${ }^{5}$ Steven Verstockt, ${ }^{1}$ Joke Schuermans. 'Department of Rehabilitation Sciences and Physiotherapy, Ghent University, Ghent, Belgium; '²Department of Movement and Sports Sciences, Ghent University, Ghent, Belgium; ${ }^{3}$ Royal Standard de Liège, Liège, Belgium; ${ }^{4}$ Zulte Waregem, Waregem, Belgium; ${ }^{5}$ Department of electronics and information systems, Ghent University, Ghent, Belgium

\subsection{6/bjsports-2021-IOC.390}

Background COVID-19 substantially impacts cardiorespiratory functioning, potentially affecting physical performance of elite athletes.

Objective To investigate the association between COVID-19 and physical performance in elite male football players.

Design A prospective cohort study during the first half of the 2020-2021 season.

Setting Belgian professional football.

Participants 84 players of three elite football teams.

Assessments Strength tests (Nordbord and Groinbar), vertical jump tests (Squat jump and Countermovement jump) and the YoYo Intermittent Recovery test - Level 1 (YYIR1) were assessed at fixed time intervals throughout the season. Polymerase chain reaction (PCR) testing was performed before each official game to detect COVID-19 infection.

Main Outcome Measurements Athletic performance was evaluated by within- and between group comparison.

Results Twenty-two subjects tested positive for COVID-19 during the follow-up period. When comparing heart rate (HR) values (normalized to the athlete's maximal HR) during YYIR1 between formerly infected players and healthy controls of the first testing after infection $(52 \pm 11.23$ days after positive PCR testing), a significantly higher HR was found in formerly infected players at 3 minutes $(p=0.017)$ and a trend towards significance was found at 6 minutes $(p=0.061)$. These in-between group differences were resolved at the second testing after infection (127.62 \pm 33.10 days after positive PCR testing). When comparing the YYIR results before and after infection within the group of infected players, trends towards significantly higher $H R$ at $3 \quad(p=0.057)$ and 6 minutes $(p=0.068)$ were seen, with no residual within group differences at the second testing after infection. Interestingly, none of the strength and vertical jump tests presented any association with COVID-19 infection.

Conclusions Intermittent aerobic endurance capacity evaluated by the YYIR1 test was established to be significantly lower in professional football players previously infected with COVID19. These decrements appeared to resolve with time. 